\title{
Presentación
}

\section{MÁS ALLÁ DE LOS PARADIGMAS: MIRADAS ACTUALES SOBRE THOMAS KUHN}

\author{
BEYOND PARADIGMS: \\ CURRENT VIEWS ON THOMAS KUHN
}

$\mathrm{H}$ a pasado casi un siglo desde el nacimiento de Thomas S. Kuhn, y más de medio desde la publicación de La estructura de las revoluciones científicas. En este último período Kuhn pasó de ser un ignoto físico de Harvard devenido historiador de la ciencia, a uno de los filósofos más influyentes y citados del siglo pasado y de lo que va del presente. A primera vista ello parece razón suficiente para ofrecer una compilación de trabajos de análisis y discusión como los que presentamos en este número de Cuadernos de filosofía. No obstante, dedicaremos esta nota a explicitar algunas motivaciones que guiaron la elaboración de este número, así como algunas coordenadas del contexto intelectual que le dio origen.

Durante el período inmediatamente posterior a la publicación de La estructura las reacciones de los filósofos fueron más bien adversas, y marcaron un cierto clima intelectual desfavorable a la propuesta de Kuhn. Hitos de esta época son las críticas de Dudley Shapere (1964) e Israel Scheffler (I967), así como los debates con Popper, Toulmin et al, en ocasión del Coloquio realizado en Londres en I965 (en Lakatos y Musgrave 1970). La situación no cambió sustantivamente en la década de los setentas, período en que el British Journal of Philosophy of Science concentró un importante caudal de artículos contrarios a Kuhn, por cuenta de autores como Jerzy Giedymin (I970), Alan Musgrave (I97I) y Harvey Siegel (1980).

Pero mientras la propuesta de Kuhn despertaba reacciones adversas entre los filósofos, se convertía en una referencia de primer orden para sociólogos, antropólogos y demás científicos sociales. Estas comunidades encontraron en la filosofía kuhniana un sentido de autopercep- 
ción disciplinar más sustantivo y más alentador que el brindado por el empirismo lógico o el racionalismo crítico. Las consecuencias del impacto de Kuhn fuera de la filosofía de la ciencia aún resultan difíciles de ponderar. Comienzan por Feyerabend (1975) quejándose de que luego de Kuhn el campo se llenó de gente que habla del método científico sin saber por qué cae una piedra, siguen con el filicidio de Kuhn (1992) al negar su herencia intelectual en el Programa fuerte de sociología de la ciencia, y terminan colocando a La estructura como el libro más citado en las ciencias sociales (Green, 20I6).

Mientras tanto Kuhn transitó las décadas de los setentas y ochentas buscando elucidar las implicaciones de la imagen de la ciencia contenida en La estructura, a través de un camino que lo condujo a reelaborar varios de sus conceptos fundamentales. Resultado de este proceso son las nociones de taxonomía y cambio de léxico, el principio de no-solapamiento y la idea de la inconmensurabilidad como resultado del surgimiento de nuevas disciplinas científicas. También en este período se enmarca el así llamado giro lingüístico de Kuhn, y los correlativos debates con Donald Davidson (1974), Hilary Putnam (I98I), y Philip Kitcher (I982).

Durante la década de los noventas se produce una explosión de literatura secundaria sobre Kuhn que poco a poco alcanza magnitudes inabordables, coincidiendo con su muerte en 1996 y la edición de El camino desde La estructura (2000). Esto se ve reforzado en 2012 con el 50 aniversario de La estructura, que deja una oleada avasallante de monográficos, libros, conferencias, números de revistas y artículos, suficientemente voluminosa como para desestimular a cualquiera que se adentre en el mundo Kuhn con pretensiones de exhaustividad bibliográfica.

Desde finales del siglo XX Kuhn se volvió un autor extremadamente popular. Su influencia se irradió por igual en los círculos de científicos sociales que lo acogieron favorablemente desde un primer momento, en los círculos de filósofos profesionales que inicialmente rechazaron sus ideas, y en las comunidades de científicos naturales que comenzaron a entender su trabajo en términos de paradigmas y comunidades científicas. Frente a la patente sobreproducción de literatura sobre Kuhn, cabe preguntarse qué razones justifican que dediquemos este número a la discusión de su obra, y que vayan más allá de sumar algo más de tinta a un río que ya de por sí es caudaloso. En lo que sigue esbozaremos algunas de ellas.

Actualmente Kuhn es un autor con fuerte presencia no solo en las discusiones técnicas de los filósofos de la ciencia, sino también en distintas áreas de las ciencias sociales, las humanidades y las ciencias natu- 
rales. Pero las discusiones y referencias sobre el pensamiento de Kuhn fuera del ámbito profesional de la filosofía todavía están mayormente volcadas a La estructura, y no pocas veces decantan en la mera cita protocolar de colocar "Kuhn I962", cuando se menciona la palabra paradigma. La figura y la obra de Kuhn han quedado en cierta forma subsumidas bajo la noción de paradigma, que hizo una fortuna intelectual no comparable a ninguna otra cosa que Kuhn haya dicho o escrito.

Como consecuencia, el resto de la obra de Kuhn ha quedado ciertamente eclipsado por la atención dispensada a La estructura, que registra un índice de citación varias veces mayor a cualquier otro texto de Kuhn. Sin negar el valor y la originalidad propias de la noción de paradigma, entendemos que esta concentración de atención en La estructura ha perjudicado el conocimiento de la filosofía kuhniana, eclipsando otros aspectos interesantes y potencialmente fértiles de su trabajo. Poner en discusión algunas de estas facetas mediante la exploración de dimensiones menos conocidas de la obra de Kuhn, fue una de las motivaciones que nos llevó a preparar este número.

Otra buena razón para volver a Kuhn es que su influencia ha disparado muy diversas interpretaciones e inspirado tendencias muy heterogéneas de comprensión de la ciencia. Sea para bien o mal, la literatura actual sobre el pensamiento kuhniano registra una diversidad de propuestas no solo enfrentadas sino en ocasiones muy lejanas entre sí, que difícilmente pueda agruparse en una imagen de conjunto. En el mundo Kuhn conviven lecturas naturalistas como las de Bird (2000), Barker, Chen y Andersen (2003), orientaciones de filosofía de la práctica científica (Rouse 2003; Chang 2012) e interpretaciones inspiradas en el estructuralismo metateórico (Lorenzano y Nudler 2012). El panorama se amplía si se toman en cuenta las lecturas neokantianas de Hoyningen-Huene (1993), Solís (1997) y Friedman (2012), la vertiente que explora las conexiones entre Kuhn y el empirismo lógico (Reisch (I99I), Irzik y Grünberg (1995) y Pinto de Oliveira (2007), y quienes como Marcum (2005) y Wray (20II) enfatizan la dimensión evolutiva de la filosofía kuhniana.

Finalmente cabe mencionar el estudio de los escritos inéditos de Kuhn, que ha tomado impulso merced a los trabajos de Marcum (2005), Hufbauer (2012), Hoyningen-Huene (2015) y Mayoral (2017). La selección de trabajos que presentamos en este número es por demás insuficiente para dar cuenta de esta riqueza de vertientes pero resulta representativa de algunas de ellas, al tiempo que provee un interesante mapa de ruta para incursionar en el intrincado paisaje de los analistas kuhnianos. 
Finalmente, un argumento no menor para sumar en la producción sobre Kuhn es la emergencia de visiones críticas, que van desde poner a debate aspectos técnicos y puntuales, hasta declarar la imagen kuhniana de la ciencia como perimida y carente de utilidad en el debate contemporáneo. Allí cabe ubicar los trabajos clásicos de Sankey (I994) y Fuller (200o), junto a los recientes colectivos de Richards y Daston (20I6) y Mizrahi (20I7).

A partir de este contexto de producción presentamos al lector los siete trabajos que componen este número, que tuvo en su concepción la idea de conjuntar textos de investigadores anglosajones e hispanohablantes, bajo la premisa de contribuir a la internacionalización de los circuitos de trabajo conjunto en torno a la obra de Kuhn.

Una obra notoria en la literatura reciente sobre Kuhn es Thomas Kuhn's Revolution: An Historical Philosophy of Science publicado en 2005, donde James Marcum ofreció una detallada reconstrucción del desarrollo intelectual de Kuhn apelando a varios textos inéditos que aún esperan publicación. En su contribución "Las filosofías de la ciencia de Thomas Kuhn: del ciclo revolucionario al árbol evolutivo”, el profesor Marcum indaga en el proceso que condujo a Kuhn desde una filosofía histórica de la ciencia a una filosofía evolutiva. Para ello efectúa un recorrido que cubre desde la concepción cíclica de las revoluciones científicas contenida en La estructura, a la imagen evolutiva del progreso por especialización, central en los últimos escritos de Kuhn. Considera Marcum que esta última constituye una descripción más adecuada de la práctica y el progreso científicos, para lo cual recurre a un estudio de caso en historia de la biología.

A modo de conclusión, Marcum reivindica el valor de la concepción evolutiva de Kuhn desde una mirada pluralista y perspectivista, "la imagen evolutiva de la ciencia de Kuhn promueve una imagen general alternativa respecto de las relaciones históricas de las diversas ciencias naturales y sus especialidades a medida que ellas evolucionan. De esta manera explica tanto el énfasis pluralista como perspectivista de la filosofía de la ciencia contemporánea al dar cuenta de las relaciones evolutivas entre las especialidades científicas en términos de sus ancestros comunes."

El artículo de Juan Vicente Mayoral, "La ruta de Kuhn hacia la discontinuidad: más allá de la historia de la ciencia”, profundiza algunos tópicos explorados por el autor en su libro Thomas S. Kuhn: la búsqueda de la estructura, publicado en 2017 y que constituye hasta hoy la más completa biografía intelectual de Kuhn editada en cualquier idioma. Afirma 
Mayoral que la imagen discontinuista de la ciencia que toma forma en La estructura se nutre de influencias tempranas provenientes de la educación universitaria de Kuhn y de su trabajo como físico. Como ya lo ha hecho en otros trabajos, el autor señala lo erróneo de la imagen de Kuhn como un historiador de la ciencia tardíamente devenido filósofo. En apoyo de esto muestra cómo varias de las preocupaciones filosóficas de Kuhn son previas a su contacto con la historia de la ciencia, y ya pueden rastrearse en las Conferencias Lowell de I95I.

La educación progresista que recibió Kuhn, su experiencia como físico en Harvard y el proceso de elaboración de las Conferencias Lowell, brindan a Mayoral el marco para indagar en los orígenes de la oposición de kuhniana a la historiografía Whig, y en sus críticas al empirismo lógico y al operacionalismo. También le permite localizar las tempranas inclinaciones de Kuhn hacia el neokantismo y el naturalismo en el período 1942-1945. En tren de balance, Mayoral apunta a integrar estas vertientes de pensamiento en las raíces historiográficas del pensamiento kuhniano, enfatizando que "[e]s imposible evaluar convenientemente su obra sin atender a la importancia que su investigación historiográfica tiene en el desarrollo de su teoría filosófica. Pero es también aconsejable atender a aquellos recursos conceptuales con los que se ocupó de manejar el abundante material historiográfico con que trató."

El texto de Leandro y Matías Giri, "Recuperando un programa kuhniano en historia de la ciencia", propone una revisión de la propuesta historiográfica de Kuhn a partir de Desarrollo científico y cambio de léxico (Kuhn 1984), la serie de conferencias Thalheimer que Kuhn dictó en I984 y que fueron recientemente editadas en español. Señalan los autores que este texto brinda una perspectiva amplia y sistemática de las reformulaciones que el pensamiento de Kuhn experimentó con posterioridad a La estructura, y desarrollan una reconstrucción enfocada en las limitaciones de la historiografía anti-whig, las bases metafísicas del pensamiento de Kuhn y los aspectos historiográficos de la dinámica del cambio científico.

A partir del papel central que la noción de léxico juega en los últimos textos de Kuhn, los autores extraen algunas conclusiones sobre el trabajo del historiador en clave kuhniana, "si bien en esencia el pensamiento kuhniano no ha variado de manera sustancial desde los tiempos de ERC hacia su etapa más refinada, sí han cambiado las prioridades analíticas y ha surgido la necesidad de profundizar varias nociones, y por ello el marco interpretativo se ha modificado drásticamente. Los historiadores que pretendan trabajar bajo este marco ya no intentarán 
mostrar la presencia de los típicos elementos de un paradigma en el episodio analizado, sino que concentrarán su arsenal analítico en la interpretación del léxico antiguo y la reconstrucción del mundo de la época a partir de él."

"Inconmensurabilidad, intraducibilidad e intensionalidad: Una discusión entre Thomas Kuhn y Donald Davidson" es el título del trabajo de Ángel Rivera-Novoa, que hace foco en el circuito de debate abierto a partir de "De la idea misma de un esquema conceptual" (1975) de Davidson y la respuesta de Kuhn en "Conmensurabilidad, comunicabilidad y comparabilidad" (1983). Tomando el riesgo de abordar un tópico sobre el que se ha escrito hasta rabiar, el texto de Rivera-Novoa abre una perspectiva interesante al afirmar que la argumentación de Davidson resiste a las respuestas ofrecidas por Kuhn basadas en la distinción entre interpretación y traducción. Para esto afirma que la versión parcial de la intraducibilidad acuñada por Kuhn no resulta exitosa, en cuanto toda interpretación supone la puesta en marcha de procesos de traducción, por lo que la distinción entre interpretar y traducir un lenguaje se torna insostenible.

Luego de una discusión que se detiene en el método davidsoniano de la interpretación radical, la noción tarskiana de verdad y los desarrollos sobre la intraducibilidad de Howard Sankey, el autor concluye que "la estrategia más adecuada para poder salvar una noción de inconmensurabilidad es tratar de hacerlo sin apelar a los fenómenos de intraducibilidad [...]. Si es posible defender la existencia de estas redes de conceptos y taxonomías sin acudir a la intraducibilidad (ni tampoco a la intensionalidad), tendríamos una noción de inconmensurabilidad que escaparía a la crítica de Davidson y que, por tanto, no se comprometería con el espinoso asunto de la intraducibilidad ni con la problemática existencia de esquemas conceptuales."

En el texto “¿Hay inconmensurabilidad tecnológica?” Daian Flórez plantea una original aplicación de la tesis de la inconmensurabilidad al desarrollo de la tecnología, mediante la noción de inconmensurabilidad tecnológica. Sostiene esta formulación en dos razones: primero, el mismo Kuhn recurrió a ejemplos de desarrollo tecnológico para apoyar la inconmensurabilidad entre teorías científicas, segundo, la propia historia de la tecnología provee soporte para afirmar la relación de inconmensurabilidad entre modelos tecnológicos. A entender de la autora, el ejemplo kuhniano del pasaje de la teoría del contacto de la pila voltaica a la teoría química de la batería constituye un caso de inconmensurabilidad tecnológica, dado por la transición entre dos formas incompatibles de explicar la fuerza electromotriz de las pilas eléctricas. A esto se suma el 
tránsito de la teoría material del calor a la termodinámica, que permite la exploración de las diferencias entre formas contrapuestas de explicar el funcionamiento de la máquina de vapor.

En ambos casos pueden detectarse términos intraducibles y variaciones semánticas directamente vinculadas al funcionamiento de artefactos -la pila voltaica y la máquina a vapor- que permiten afirmar la inconmensurabilidad entre teorías tecnológicas. Esto permite a Flórez sostener que "hay inconmensurabilidad tecnológica no obstante la resistencia que se podría derivar de la idea de que la inconmensurabilidad como fenómeno semántico no es atribuible a los artefactos. Un razonamiento como ese proviene de la identificación equivocada de la tecnología con los artefactos, y en la medida en que explico la naturaleza errónea de dicha identificación, considero que se puede rechazar dicha línea de análisis."

El trabajo "A pluralist interpretation of Thomas Kuhn's philosophical position" escrito por Paula Atencia Conde-Pumpido, se centra en las implicanciones metafísicas del pensamiento de Kuhn, para ensayar una lectura pluralista de la tesis de la inconmensurabilidad. Entiende Atencia que la inconmensurabilidad puede ser leída en clave pluralista como implicando divergencias taxonómicas y ontológicas entre especialidades sucesivas o teorías rivales. Para esto desarrolla una estrategia argumental basada en la premisa de que el mundo no se compone de una serie predefinida de objetos y clases, lo cual habilita formas alternativas de organizar la experiencia.

La autora enfatiza que para el último Kuhn las taxonomías constituyen modos fundamentales de organización de la experiencia, en cuanto definen los individuos y relaciones de clases que son posibles en un ordenamiento dado. Cada taxonomía delimita una ontología, y la existencia de múltiples taxonomías permite postular múltiples ontologías correlativas, punto clave para el pluralismo metafísico que Atencia atribuye a Kuhn. Estos términos permiten entender a la inconmensurabilidad como la tesis de que dos teorías que se enfrentan durante una revolución científica disienten tanto en términos ontológicos como taxonómicos, lo que permite afirmar que "[l]as especialidades sucesivas o rivales son inconmensurables porque asumen estructuras taxonómicas no-homólogas, esto es, taxonomías que emplean relaciones incompatibles de similaridad y disimilaridad. Como resultado, las especialidades inconmensurables también presuponen ontologías disidentes."

Alexander Bird se convirtió en un referente en los estudios kuhnianos a partir de la publicación del sugestivo Thomas Kuhn en el año 2000. 
En los últimos tiempos los intereses de Alexander se han concentrado en temas de epistemología y filosofía de la medicina, por lo que es un honor que vuelva a incursionar en el mundo Kuhn para contribuir con este monográfico. En su artículo "Comprendiendo los paradigmas de Kuhn a la luz de la ciencia cognitiva", defiende Bird que la noción de paradigma se ve reforzada por desarrollos recientes en psicología y ciencia cognitiva. Considera que la idea kuhniana del trabajo paradigmático centrado en ejemplares involucra una teoría de la cognición científica, para la cual Kuhn se nutrió de avances de la psicología empírica.

Luego de un examen pormenorizado de la teoría kuhniana de los ejemplares y su papel en el aprendizaje científico, Bird postula que la imagen de la cognición científica basada en ejemplares tiene consecuencias directas en los problemas de la racionalidad científica: "Los filósofos contemporáneos a Kuhn exigieron un vínculo estrecho entre cognición, lógica y racionalidad. Sin embargo, muchos procesos cognitivos no pueden reducirse a la lógica, por ejemplo, el reconocimiento de patrones. Pero eso no los hace irracionales. Aquí se puede tomar una decisión semántica: cualquiera puede decir que Kuhn ha demostrado que la ciencia cognitiva exitosa involucra centralmente procesos cognitivos no racionales (pero no irracionales); o se puede decir que Kuhn ha demostrado que la racionalidad de la ciencia implica desplegar, además de procesos de pensamiento lógico, otros procesos de pensamiento que no pueden reducirse a la lógica."

Los trabajos que componen este número constituyen un conjunto sumamente heterogéneo pero a la vez representativo del estado actual de los estudios sobre Kuhn. En nombre de Cuadernos de filosofía queremos agradecer a cada uno de los autores por su generosa contribución y su comprensión de los tiempos editoriales. Esperamos que los trabajos aquí incluidos resulten útiles y estimulantes tanto para investigadores familiarizados con la filosofía de Kuhn como para estudiantes de grado y posgrado con intereses en filosofía de la ciencia.

Pablo Melogno

Montevideo, julio de 2020 


\section{Referencias bibliográficas}

Barker, P., Chen, X., Andersen, H. (2003). "Kuhn on Concepts and Categorization". En T. Nickles, 2I2-245.

Bird, A. (2000). Thomas Kuhn. Madrid: Tecnos, 2002.

Chang, H. (2012). Is Water $\mathrm{H}_{2} \mathrm{O}$ ? Evidence, Realism and Pluralism. BSPS, 293. Dordrecht: Springer.

Davidson, D. (1974). "De la idea misma de un esquema conceptual". En De la verdad y de la interpretación, I89-203. Barcelona: Gedisa, 1990.

Feyerabend, P. (198I [1975]). "Cómo defender a la sociedad contra la ciencia”. En I. Hacking (comp.), Revoluciones científicas, 294-3I4. México: FCE.

Friedman, M. (20I2). "Kuhn and Philosophy". Modern Intellectual History 9 (I): $77-88$.

Fuller, S. (2000). Thomas Kuhn: A Philosophical History for Our Times. Chicago: Chicago University Press.

Giedymin, J. (1970). "The paradox of meaning variance". British Journal for the Philosophy of Science 2I: 257-268.

Green, E. (2016). "What are the Most-Cited Publications in the Social Sciences (According to Google Scholar)?." Disponible en http://blogs.lse.ac.uk/impactofsocialsciences/2016/05/12/what-arethe-most-cited-publications-in-the-social-sciences-according-togoogle-scholar/ [Consulta: 08/07/2020].

Hoyningen-Huene, P. (1993). Reconstructing Scientific Revolutions. Chicago: The University of Chicago Press.

- (2015). "Kuhn's Development Before and After Structure". En W. Devlin, A. Bokulich (eds.). Kuhn's Structure of Scientific Revolutions 50 years on, BSPS, 3II, I85-195. Switzerland: Springer.

Hufbauer, K. (20I2). "From Student of Physics to Historian of Science: T. S. Kuhn's Education and Early Career, 1940-1958". Physics in Perspective I4: 42I- 470.

Irzik, G.; Grünberg, T. (1995). "Carnap and Kuhn: Arch Enemies or Closed Allies?”. British Journal of Philosophy of Science 46: 285-307.

Kuhn, T. (2013 [1962]). La estructura de las revoluciones científicas. México: FCE.

(1983). "Conmensurabilidad, comparabilidad, y comunicabilidad”. En Kuhn, T. ¿Qué son las revoluciones científicas?, 95-I35. Barcelona: Paidós, 1989. 
(2017 [1984]). Desarrollo científico y cambio de léxico. Montevideo: ANII/FIC/SADAF. Edición crítica de Pablo Melogno y Hernán Miguel.

- (1992). "El problema con la filosofía de la ciencia histórica". En El camino desde La estructura, I3I-I48. (2000). El camino desde La estructura. Barcelona: Paidós, 2002.

Kitcher, P. (1982). "Implications of Incommensurability". PSA, Proceedings of the Biennial Meeting of the Philosophy of Science Association, v. 2: Symposia and Invited Papers: 689-703.

Lakatos, I., Musgrave, A. (eds.) (I975 [1970]). La crítica y el desarrollo del conocimiento. Barcelona: Grijalbo.

Lorenzano, P., Nudler, O. (2012). El camino desde Kuhn. La inconmensurabilidad hoy. Madrid: Biblioteca Nueva.

Marcum, J. (2005). Thomas Kuhn's Revolution: An Historical Philosophy Of Science. New York: Continuum.

Mayoral, J. (20I7). Thomas S. Kuhn: la búsqueda de la estructura. Zaragoza: Prensas de la Universidad de Zaragoza.

Mizrahi, M. (ed.) (2017). The Kuhnian Image of Science. Time for a Decisive Transformation? London: Rowman \& Littlefield.

Musgrave, A. (197I). "Kuhn's second thoughts". British Journal for the Philosophy of Science 22: 287-297.

Nickles, T. (ed.) (2003). Thomas Kuhn. Contemporary Philosophy in Focus. Cambridge-UK: Cambridge University Pres.

Putnam, H. (I988 [198I]). Razón, verdad e historia. Madrid: Tecnos.

Pinto de Oliveira, J.C. (2007). "Carnap, Kuhn, and revisionism: on the publication of Structure in Encyclopedia". Journal of General Philosophy of Science 38: I47-I57.

Reisch, G. (199I). “Did Kuhn Kill Logical Empiricism?". Philosophy of Science 58 (2): 264-277.

Richards, R., Daston, L. (eds.) (2016). Kuhn's Structure of Scientific Revolutions at Fifty: Reflections on a Science Classic. Chicago: University of Chicago Press.

Rouse, J. (2003). "Kuhn's Philosophy of Scientific Practice". En T. Nickles, IOI-I2I.

Sankey, H. (1994). The Incommensurability Thesis. Aldershot: Avebury.

Scheffler, I. (1967). Science and Subjectivity. Indianapolis: Bobbs-Merrill.

Shapere, D. (1964). "The Structure of Scientific Revolutions". The Philosophical Review LXXIII: 383-394.

Siegel, H. (1980). "Objectivity, Rationality, Incommensurability, and more". British Journal for the Philosophy of Science 3I (4): 359-375. 
Más allá de los paradigmas: miradas actuales sobre Thomas Kuhn | P. Melogno

Solís, C. (1997). "La revolución kantiana de Kuhn". Endoxa 9: 5-30.

Wray, B. (20II). Kuhn's evolutionary social epistemology. Cambridge-UK: Cambridge University Press. 